\title{
Parasitosis intestinal y alternativas de disposición de excreta en municipios de alta marginalidad
}

\author{
Intestinal parasitosis and feces excretion alternatives in \\ municipalities characterized by high marginality
}

\author{
María Teresa Guerrero Hernández'; Yolanda Hernández Molinar'; María \\ Eugenia Rada Espinosa" ; Álvaro Aranda Gámez "I; Martha I nés Hernández ${ }^{\text {IV }}$ \\ 'Máster en Salud Pública. Universidad Autónoma de San Luis de Potosi, México. \\ "Máster en Antropología Cultual. Universidad Autónoma de San Luis de Potosi, \\ México. \\ II"QFB. Profesor hora clase. Universidad Autónoma de San Luis de Potosi, México. \\ IV Máster en Ciencias Básicas. Universidad Autónoma de San Luis de Potosi, México.
}

\section{RESUMEN}

I ntroducción Las enfermedades diarreicas y las parasitosis intestinales siguen constituyendo un problema de salud pública en el mundo, los niños son los más vulnerables. En el entorno rural de México, el hacinamiento, la escasez de agua potable y carencia de drenaje, han generado un deterioro permanente del estado de salud de la población, que se agudiza en zonas de alta marginalidad.

Objetivos Identificar la prevalencia de parasitosis intestinal y las alternativas tecnológicas de disposición de excretas, en nueve municipios de alta marginalidad. Métodos Se realizó un estudio coproparasitoscópico a 621 niños en edad escolar, las muestras fueron procesadas por el método de kato- kats para la búsqueda de helmintos y se analizaron las condiciones de saneamiento básico, vivienda, presencia de diarrea y expulsión de parásitos intestinales en los dos últimos meses. Resultados La prevalencia de parasitosis intestinal fue de $30 \%$. El 22,3\% dio positivo a protozoarios y el $7,9 \%$ a geohelmintos. En protozoarios los más frecuentes fueron la Entamoeba histolytica con 10,5\% y la Giardia lamblia con 8, 1 . Entre los geohelmintos, el Ascaris lumbricoides con 6,0\%. La mitad de las madres de los niños eran analfabetas, $81,4 \%$ de los padres, agricultores y jornaleros; 46,7 $\%$ vivía en casas de uno o dos cuartos, el $40 \%$ de las viviendas tenía techo de palma y el $63 \%$ piso de tierra. El 75,7 \%, de los niños expulsaron parásitos y uno de cada tres niños refirió que al menos un hermano también expulsó parásitos en el mismo período. Del total de niños que presentaron diarrea el 68,8 \% disponía su 
excreta en letrinas tradicionales y el 5,6 \%, en sanitarios ecológicos secos. Conclusiones La prevalencia de parasitosis intestinal en municipios de alta marginalidad es consistente con las condiciones de pobreza y carencia de servicios básicos.

Palabras clave: Parasitosis intestinal, disposición de excretas, saneamiento básico, sanitario ecológico seco.

\section{ABSTRACT}

Introduction Diarrheal diseases and intestinal parasitosis are still public health problems worldwide, being the children the most vulnerable. In the Mexican rural setting, crowded conditions, shortage of drinking water and of appropriate drainage system have deteriorated in a permanent way the population's health status, a situation that becomes more acute in high marginality areas.

Objectives To identify the prevalence of intestinal parasitosis and the technological alternatives of feces excretion in 9 municipalities with high marginality index.

Methods A coproparasitoscopic study was conducted in 621 school children; the samples were examined by kato-kats method for helminths. Basic sanitary conditions, dwelling, incidence of diarrheas and excretion of intestinal parasites during the last two months were studied.

Results Intestinal parasitosis incidence was $30 \%, 22.3 \%$ was positive to protozoos and $7.9 \%$ to geohelmints. The most common protozoa were entamoeba histolytica accounting for $10.5 \%$ and Giardia lamblia for $8.1 \%$. Among geohelmints, Ascaris lumbricoides with $6.0 \%$. Half of these children's mothers were illiterate; $81.4 \%$ of parents were farmers and labourers, $46.7 \%$ lived in one or two-room houses, $40 \%$ of houses had palm roof and $63 \%$ had their floor made of soil. Of the total number of studied children, $75.7 \%$ had excreted parasites and one out of three stated that at least one sibling had also excreted parasites in the same period of time. Of the total amount of children presented with diarrheas, 68.8 $\%$ defecated in traditional latrines and $5.6 \%$ did in dry ecological toilets.

Conclusions Prevalence of intestinal parasitosis in municipalities with high marginality index resulted from poverty conditions and the lack of basic services.

Key words: Intestinal parasitosis, feces excretion, basic sanitation, dry ecological toilet.

\section{NTRODUCCI ÓN}

Las enfermedades diarreicas y parasitosis intestinales siguen constituyendo un problema de salud pública en el mundo, los niños son los más vulnerables. En México ocupan uno de los primeros lugares como causas de morbilidad y mortalidad en niños menores de cinco años. En el Instituto Mexicano del Seguro Social (IMSS) las helmintiasis constituyen la sexta causa de demanda de consulta, y en el grupo de 1 a15 años es el tercer motivo de consulta. ${ }^{1}$ 
Las comunidades que carecen de infraestructura de saneamiento básico, enfrentan la contaminación biológica y sus consecuencias, por ende la provisión de sistemas de disposición de excreta es esencial en el control de parasitosis intestinal, ya que los agentes patógenos expulsados con las heces fecales pueden sobrevivir en suelo y estanques el tiempo suficiente para representar un riesgo potencial para la salud de la población. ${ }^{2}$

La relación entre saneamiento deficiente e infecciones gastrointestinales, al igual que la ausencia de drenaje intradomiciliar y mortalidad por infecciones gastrointestinales han sido ampliamente documentadas a nivel clínico y epidemiológico, por lo anterior, el hacinamiento, la falta de agua potable y de sistemas de disposición de excreta, así como la contaminación de alimentos y agua son variables presentes en el entorno rural en México, que han propiciado y acumulado un deterioro permanente del estado de salud de la población . ${ }^{3}$

En este contexto la parasitosis intestinal se agudiza en zonas de alta marginalidad en donde las condiciones de vivienda, higiene personal y educación son precarias, situación que demuestra y exige la importancia del entorno en la promoción de salud, ya que si este no es apropiado, repercutirá en la biología humana, el estilo de vida y en la organización de la atención sanitaria.

\section{MÉTODOS}

El presente estudio es descriptivo y retrospectivo. El universo estuvo constituido por la población escolar de centros de educación primaria en la zona rural de la Huasteca, de la cual se obtuvo una muestra estratificada; primero se seleccionaron nueve municipios de alta marginalidad, después las localidades, y finalmente las escuelas y en estas al total de niños que cursaban de primero a cuarto grado. La muestra final fue de 621 niños.

Este estudio se desarrolló en dos etapas: en la primera etapa se aplicó un cuestionario para obtener información general sobre características socioeconómicas, abastecimiento y tratamiento de agua, sistemas de disposición de excretas, condiciones de la vivienda, presencia de diarrea, signos y síntomas de parasitosis intestinal en los últimos dos meses. En la segunda etapa a cada niño se le realizó un seriado de tres muestras que fueron procesadas a través de la técnica de Kato-Katz para la búsqueda de helmintos. El día anterior a la toma de la muestra, se proporcionó a cada niño un recipiente de cierre hermético, limpio, etiquetado con el nombre del niño, escuela, grado escolar, grupo, localidad y municipio. Para la recolección de la muestra se orientó a las madres y niños que depositaran la materia fecal en el recipiente, que se colocó en una bolsa plástica y guardó en el lugar más fresco de la casa. Las muestras se recibieron y guardaron en cajas de unicel con refrigerante y posteriormente fueron trasladadas al laboratorio.

En asambleas de padres de familia se informó sobre las actividades del presente estudio, y pasos para la toma de muestras de heces fecales para el examen coproparasitoscópico, que se realizó previo consentimiento de las madres de los niños objeto de estudio.

\section{RESULTADOS}


Las poblaciones se caracterizaron porque el $53 \%$ de las viviendas tenía las paredes de otate, el $40 \%$ techo de palma y el $63 \%$ piso de tierra. El $54 \%$ de las familias estaban integradas por 5 a 7 miembros y el $24 \%$ por 8 a 10 miembros, finalmente, el $21 \%$ de las madres de los niños eran analfabetas y el $32 \%$ tenía primaria incompleta o no rebasaba el tercer grado de enseñanza básica.

Del total de niños, el 51,1 \% eran varones, el 48,9\% hembras y el 76,9 \% tenía entre 6 y 9 años de edad. Del total de muestras el $30 \%$ resultaron positivas. En los protozoarios (22,3\%), las formas parasitarias de mayor frecuencia fueron la Entamoeba histolytica con el 10,5\%, y la Giardia lamblia con el 8,1\%. En los geohelmintos (7,9\%) la forma parasitaria más frecuente fue el Ascaris lumbricoides con el 6,0 \%, el Entorobius vermicularis, Necator americanus, Uncinaria y Strongyloides (1\%) tuvieron una menor prevalencia (tabla).

En relación con el entorno rural y la parasitosis intestinal, ( fiq.1) los niños que disponían sus excretas en letrina convencional mostraron la mayor prevalencia de protozoarios y helmintos. Entre los niños que la disponían en sanitarios ecológicos secos, se presentó un caso de Strongyloides stercolaris.

El $21,9 \%$ de los niños expulsó parásitos en el período de agosto a octubre y el 28,4 $\%$ de los hermanos, también lo hizo en el mismo período. Se registró que a menor edad, mayor fue la frecuencia de expulsión de parásitos (fig. 2).

En relación con los sistemas de disposición de excreta y expulsión de parásitos, la mayor proporción ( $75 \%$ ) de los niños que expulsaron parásitos contaban con letrina tradicional y la menor proporción se presentó en los niños que contaban con sanitario ecológico seco (fig. 3 ). El sanitario ecológico seco es una alternativa tecnológica de caída y deposito, basa su funcionamiento en la separación de excreta y orina y evita el contacto de la excreta con suelo y agua a través de su confinamiento y biodegradación en cámaras de aislamiento. Del total de niños que tuvieron diarrea, el 68,8 \% disponía la excreta en letrinas tradicionales y el 5,6\%, en sanitarios ecológicos secos (fig. 4).

\section{DISCUSIÓN}

Los resultados mostraron una prevalencia global de parasitosis intestinal (30 \%), dos veces menor que la obtenida en estudios realizados en Oaxaca ${ }^{4}$ y Veracruz. ${ }^{5}$ Predominaron los protozoarios y específicamente Entamoeba histolytica, Giardia Lamblia y Entamoeba coli. La prevalencia de Giardia Lamblia fue similar a la registrada en un estudio realizado en Perú ${ }^{6}$ pero en relación a Entamoeba coli está muy por debajo de la obtenida en el mismo estudio. La infección por comensales como la Entamoeba coli está indicando un elevado índice de contaminación fecal en la población, y del agua para el consumo humano, posiblemente por su inadecuado almacenamiento, manipulación y tratamiento.

En relación con los geohelmintos, el Ascaris Lumbricoides se encontró con mayor frecuencia $(6 \%)$, la prevalencia fue dos veces menor a la informada en un estudio realizado en Venezuela ${ }^{7}$ y dos veces mayor que la notificada por la Secretaria de Salud en San Luis Potosí, diez años atrás ${ }^{8}$ la prevalencia de Enterobius vermicularis, Necator americanus, strongyloides stercoralis y Trichuris trichiura era menor que la reportada en el mismo estudio. 
En este trabajo se destaca que el tipo de alternativa para la disposición de excreta es debido a la iniquidad y pobreza, que se materializa esencialmente en la falta de servicios básicos en áreas de alta marginación. El hallazgo de protozoarios no patógenos es indicador de un elevado nivel de contaminación fecal en la población y del riesgo potencial de ingerir otras formas parasitarias. ${ }^{9}$

Las parasitosis siguen constituyendo una contradicción en el campo de la salud pública, sobre todo en los países en desarrollo, porque son reflejo de condiciones marginales, hábitos higiénicos deficientes, insalubridad y hacinamiento. Por tanto, el enfoque de promoción de salud en el desarrollo de la tecnología apropiada para la disposición de excretas constituye una premisa central en la lucha contra las enfermedades relacionadas con la excreta. ${ }^{10}$

La prevalencia de parasitosis intestinal en los municipios de alta marginalidad es consistente con las condiciones de pobreza y de carencia de servicios básicos. Por ende, el enfoque de promoción de salud en el diseño de alternativas tecnológicas para la disposición de excreta en áreas marginadas es una exigencia que junto con el fomento de hábitos higiénico-sanitarios pueden limitar en más de $60 \%$ la ocurrencia de diarreas y otras enfermedades relacionadas con la excreta. ${ }^{11}$

En este contexto la influencia y transformación del entorno, es decir, la purificación del agua, ${ }^{12}$ el drenaje o un sistema sanitario seguro, disminuirían las infecciones gastrointestinales y parasitarias a menos costo que las intervenciones de salud a nivel individual. ${ }^{13}$

La prevalencia de parasitosis intestinal es consistente con las condiciones de pobreza y con el tipo de alternativa tecnológica de disposición de excreta, en este caso la letrina convencional como principal sistema y fuente de contaminación fecal por filtración de heces a fuentes de abastecimiento de agua en las localidades de alta marginalidad, por ende áreas endémicas de parasitosis.

\section{REFERENCI AS BI BLI OGRÁFICAS}

1. Sistema Nacional de Información en Salud. Diez Principales causas de mortalidad en edad preescolar del 2005. Indicadores de salud [serie en Internet]. [citado 8 May 2007]. Disponible en: http: sinais.salud.gob.mx [México: SSA; 2000-2004].

2. Duncan M, Cairncross S. Aspectos de salud pública. En: OMS, editor. Directrices para el uso sin riesgos de aguas residuales y excretas en agricultura y acuicultura. España: OMS; 1990.p.65-105.

3. López Acuña D. La salud desigual en México. 9na ed. México: Siglo XXI; 1993.

4. Navarrete EJ, Navarrete CE, Escandón RC, Escobedo J. Prevalencia de parasitosis intestinal en la población infantil en Santiago Jamiltepec, Oaxaca. Rev Méd IMSS (Méx). 1993; 31:157-61.

5. Flisser A, Reynoso O, Ambrosio J. Identificación y tratamiento de parasitosis intestinales en la población de Coapeche, Veracruz. Rev Fac Med UNAM. 2002; 45(1) 14-6.

6. Cabrera SM, Vesrategui M, Cabrera R. Prevalencia de enteroparasitosis en una comunidad altoandina de la Provincia de Víctor Fajardo, Ayacucho, Perú. Rev 
Gastroenterol (Perú) [serie en Internet]. [citado 8 May 2007]. Disponible en: http://www. scielo.org.pe/scielo.php? ISSN 1022-5129.

7. Devera R, Cermeño J R, Blanco Y, Morales MC, Guerra X, Sousa M, et al. Prevalencia de blastocistosis y otras parasitosis intestinales en una comunidad rural del Estado Anzoátegui, Venezuela [serie en Internet]. [citado 8 May 2007]. Disponible en: http://www.scielo.cl/scielo.php?

8. Secretaria de Salud. Quinta encuesta centinela de helmintiasis en Ebano, San Luis Potosí. 1995. San Luis Potosí, México: Secretaria de Salud; 1995.

9. Sánchez VJ, Tay-Zavaka J, Robert GL, Romero CL, Ruiz SD, Rivas GC. Frecuencia de parasitosis intestinales en asentamientos humanos irregulares. Rev Fac Med UNAM. 2000;43 (3): 80-3.

10. Guerrero HT, Fritche TJ, Martínez ZR, Hernández MY. Diseño y construcción de sanitarios ecológicos secos en áreas rurales. Rev Cubana salud Públlica. [serie en Internet]. 2006[citado 8 May 2007]; 32(3). Disponible en: http://bvs. sld.cu/revistas/spu/vol32 3 06/spu16306. htm.

11. Almedom AM, Blumenthal U, Manderson L. Procedimientos para la evaluación de la higiene: enfoques y métodos para evaluar prácticas de higiene relacionadas con el agua y saneamiento. Lima: Centro Panamericano de Ingeniería Sanitaria y Ciencias del Ambiente (CEPIS), OPS / OMS; 1997.

12. Buck C. Después de Lalonde: La creación de la salud. En: OPS, editor. Promoción de la salud: una antología. Washington, D.C.: OPS; 1996.p.6-14. (Publicación científica No. 557).

13. McKinlay JB. Promoción de la salud por medio de políticas públicas saludables: Conceptos de la promoción de la salud: la contribución de los métodos complementarios de investigación. En: OPS, editor. Promoción de la salud: una antología. Washington,D.C.: OPS; 1996.p.60-74. (Publicación científica No. 557).

Recibido: 18 de septiembre de 2007.

Aprobado: 23 de octubre de 2007.

María Teresa Guerrero Hernández. Universidad Autónoma de San Luis Potosí (UASLP). Anahuac No. 215. Colonia Tequisquiapam. C.P. 78230. San Luis Potosí, México. Tel. (444) 81102 68. E-mails: tguerrer@uas/p.mx 
Tabla. Prevalencia de formas parasitarias intestinales en áreas rurales San Luis Potosí, México, 2005

\begin{tabular}{|c|c|c|}
\hline Formas parasitarias & Frecuencia & Porcentaje \\
\hline $\begin{array}{l}\text { Quistes de Entamoeba Histolytica } \\
\text { Quistes de Giardia lamblia } \\
\text { Quistes de Entamoeba coli } \\
\text { Huevos de Enterobius vermicularis } \\
\text { Huevos de Necator Americanus } \\
\text { Huevos de Ascaris lumbricoides } \\
\text { Huevos de Trichuris trichiura } \\
\text { Huevos de Uncinaria } \\
\text { Larvas Strongyloides stercolaris } \\
\text { Negativos }\end{array}$ & $\begin{array}{c}65 \\
50 \\
23 \\
3 \\
1 \\
37 \\
3 \\
3 \\
1 \\
435\end{array}$ & $\begin{array}{l}10,5 \\
8,1 \\
3,7 \\
, 5 \\
, 2 \\
6,0 \\
, 5 \\
, 5 \\
, 2 \\
70,0\end{array}$ \\
\hline Total & 621 & 100 \\
\hline
\end{tabular}

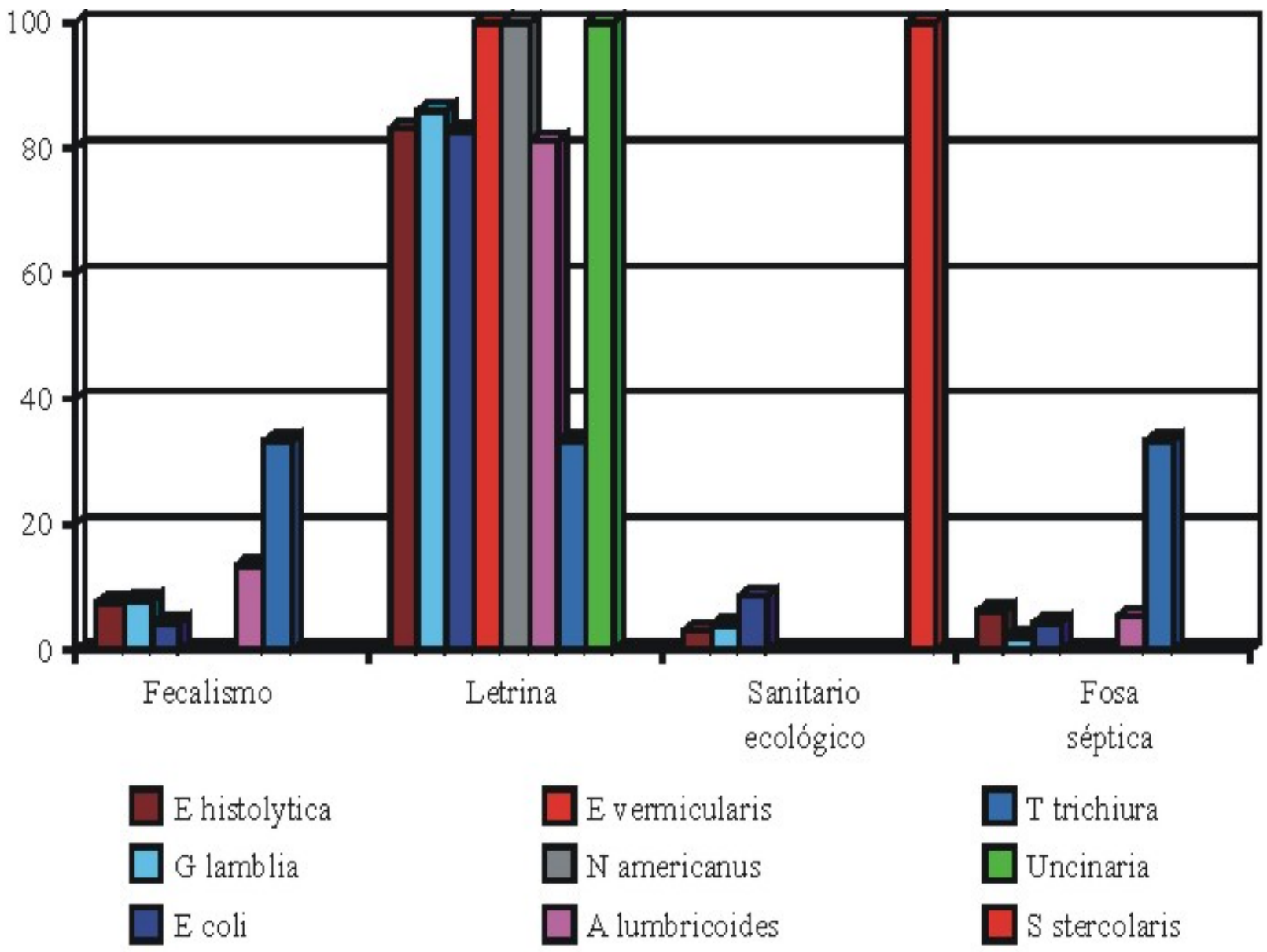

Fig.1. Formas parasitarias según alternativas de disposición de excreta. San Luís de Potosí, México 2005. 


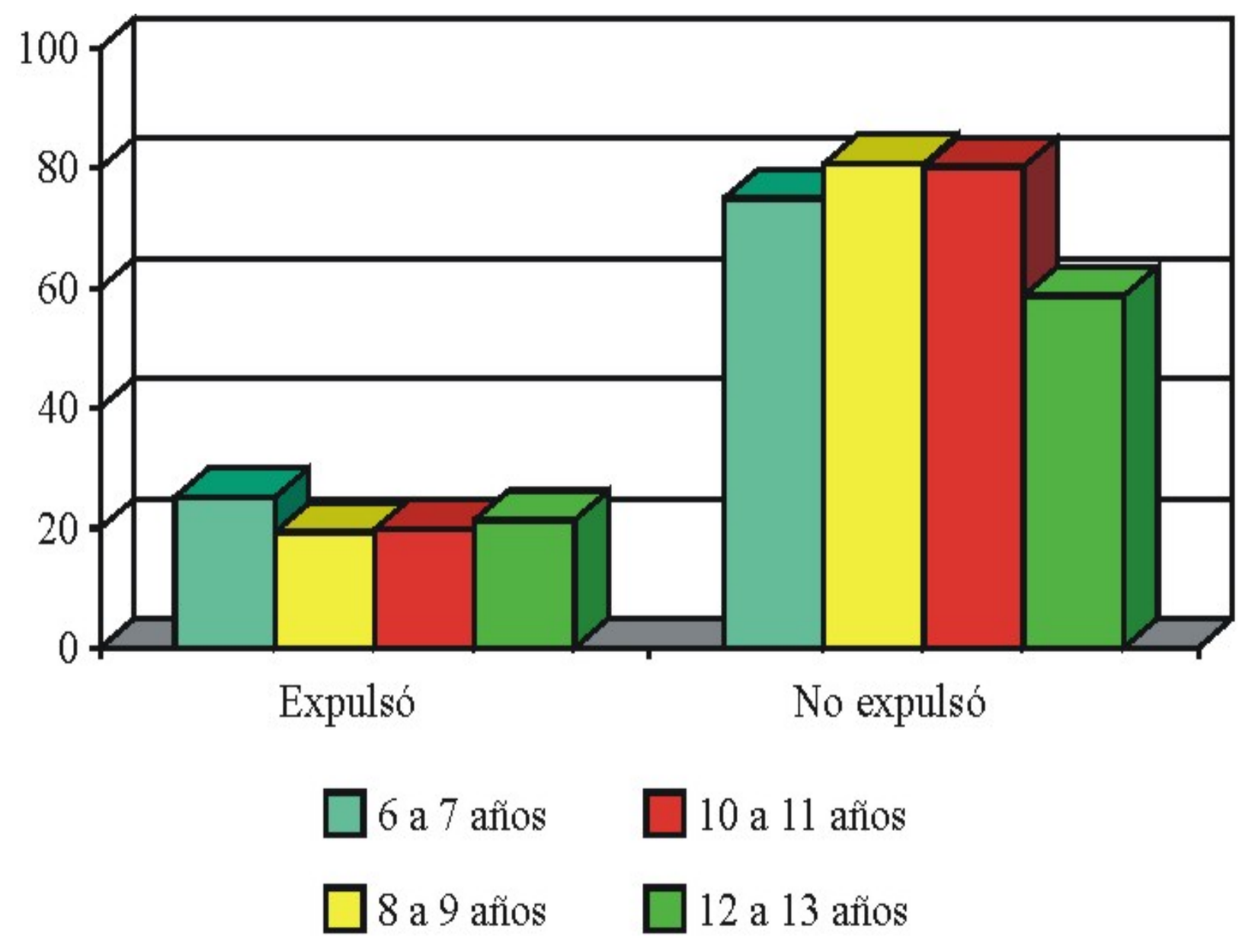

Fig.2. Expulsión de parásitos según edad. San Luís de Potosí, México 2005. 


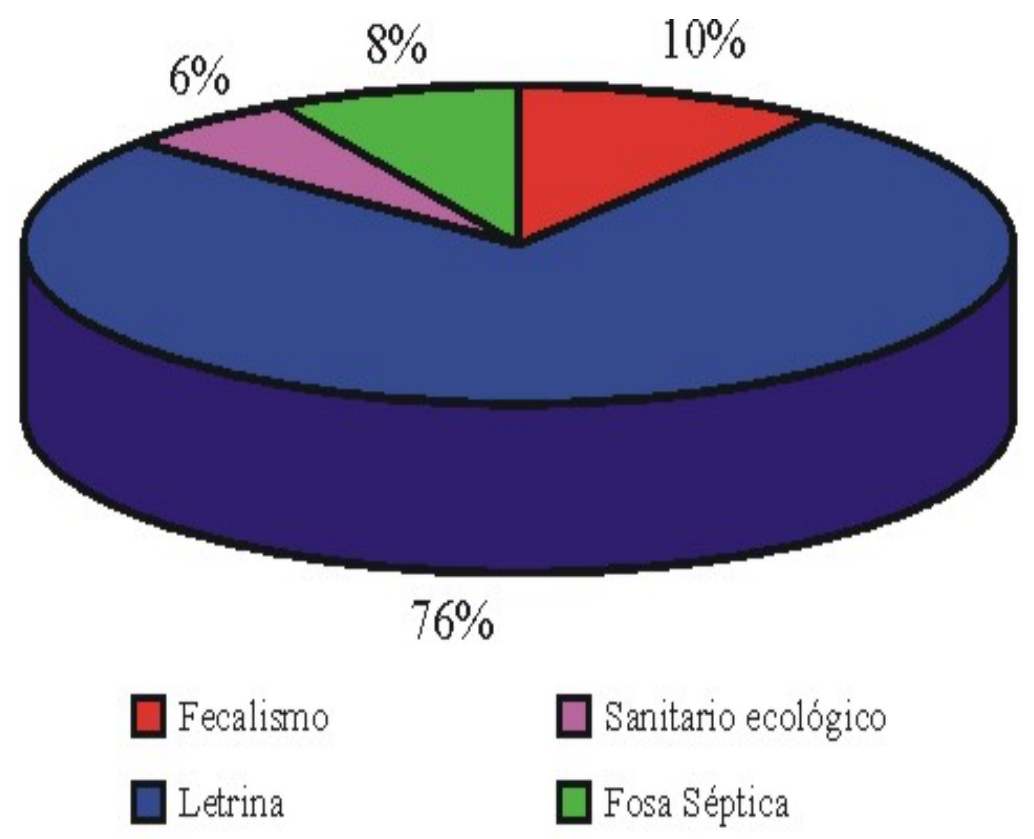

Fig.3. Expulsión de parásitos según alternativa de disposición de excreta. San Luís de Potosí, México 2005.

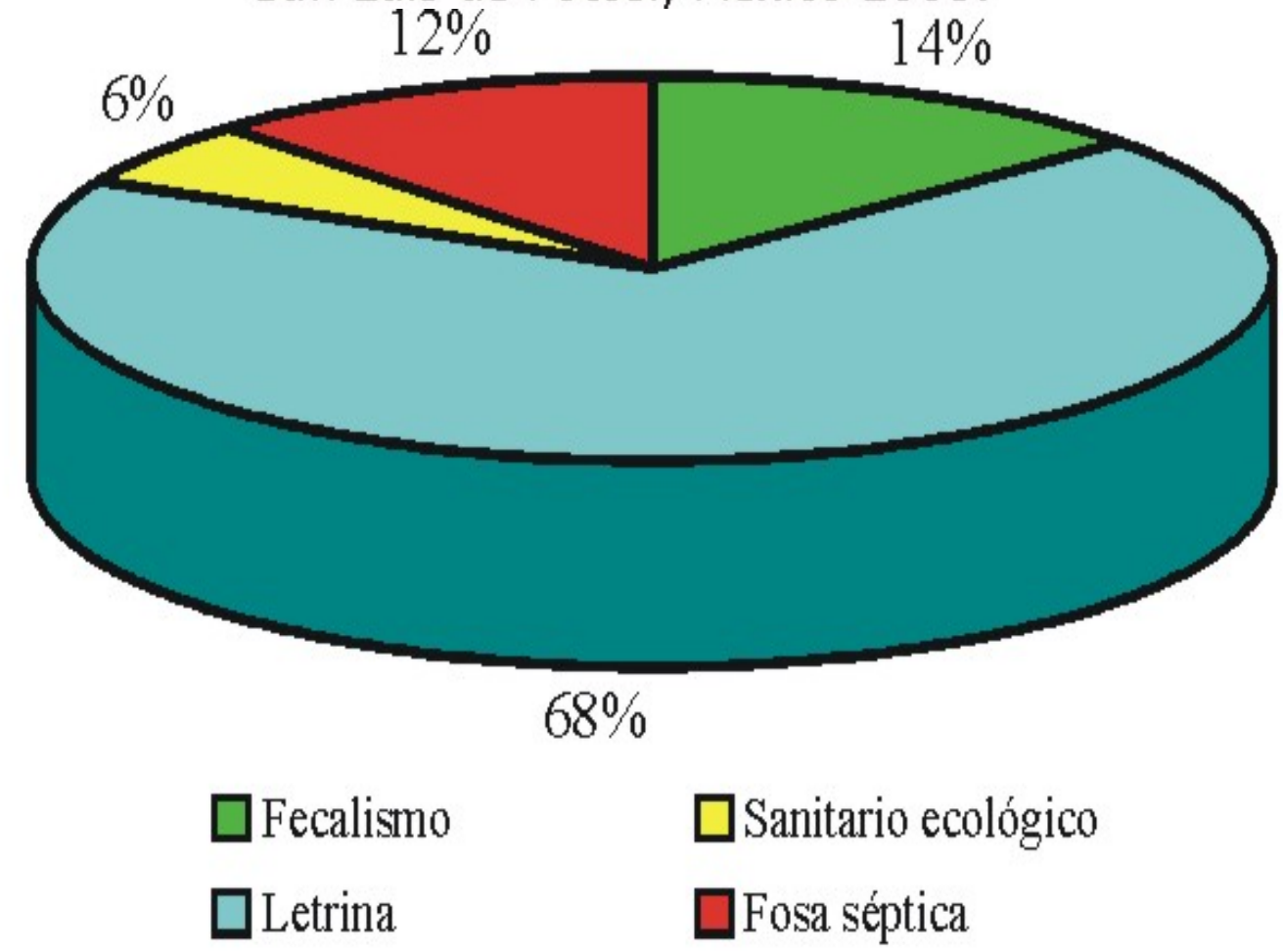

Fig.4. Diarreas según alternativa de disposición de excreta. San Luís de Potosí, México 2005. 
